\title{
High Energy Gamma Rays and Neutrinos from Star-forming Activities in the Galactic and Extragalactic Sources
}

\author{
Soebur Razzaque \\ Department of Physics, University of Johannesburg, \\ PO Box 524, Auckland Park 2006, South Africa \\ email: srazzaque@uj.ac.za
}

\begin{abstract}
The origin of the IceCube astrophysical neutrinos is an outstanding question. Starforming activities which can accelerate particles to very high energies have been suggested as possible origin of these neutrinos. I will present a scenario where a subset of the neutrino events originate from the Galactic center region and Fermi Bubbles, resulting from star-forming activities. Multi-messenger signal in high energy gamma rays and neutrinos can probe this scenario. I will also present an analysis of the statistical association of the star-forming sources in our Galaxy and outside, with astrophysical neutrinos, as well as expected neutrino signal from these sources by fitting gamma-ray data.
\end{abstract}

Keywords. neutrinos, gamma rays: theory, Galaxy: center

\section{Introduction}

The origin of Astrophysical neutrinos, so called High Energy Starting Events (HESE), detected by IceCube (Aartsen et al. 2014) is currently a hot topic for debate. The published 4-year HESE sample contains 54 neutrinos with energy between $\sim 20 \mathrm{TeV}$ and $\sim 2 \mathrm{PeV}$ (Kopper 2015). It has been argued that a subset of these HESE neutrinos are of Galactic origin (Razzaque 2013). Extragalactic sources, such as the sources of ultrahighenergy cosmic rays (Moharana \& Razzaque 2015) and star-forming objects (Emig et al. 2015) have also been used to study angular correlation with HESE neutrinos. Fermi bubbles at the Galactic centre ( $\mathrm{Su}$ et al. 2010) may produce high energy neutrinos (Crocker \& Aharonian 2011; Lunardini \& Razzaque 2012) and some HESE neutrinos might be from the Fermi bubbles (Lunardini et al. 2014).

Here we present results from our recent studies about very high-energy gamma-ray emission from the Fermi bubbles, which can be detected with High Altitude Water Cherenkov (HAWC) detector (Goodman 2016), that may constrain the hadronic model, together with neutrino detection (Lunardini et al. 2015). We also present results from our study of angular correlations of HESE neutrinos with Galactic and extragalactic sources related to star formation (Moharana \& Razzaque 2016).

\section{Results}

We find that by modelling the $\gamma$-ray emission of the Fermi bubbles (Ackermann et al. 2014 ) to $100 \mathrm{TeV}$, HAWC will be complementary to neutrino searches, realising true multi-messenger astronomy with overlapping energy ranges. It will be possible to constrain the hadronic $\gamma$-ray emission, in particular the spectral features of the cosmic rays, within a few years. A negative result by IceCube will be compatible with either a hadronic scenario with sub-PeV cutoff, or a primary leptonic origin of the Fermi bubbles. 

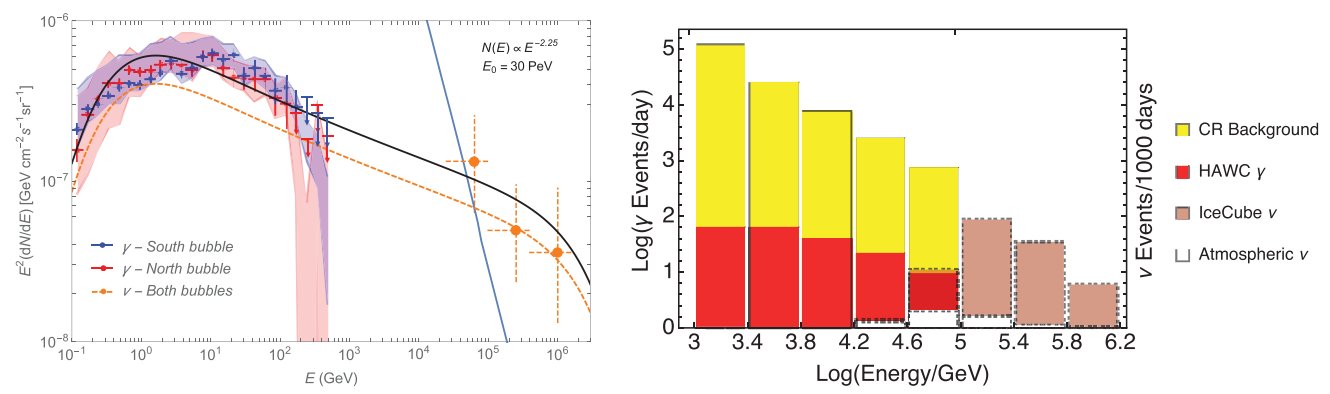

Figure 1. Left panel: Hadronic model for $\gamma$-ray and neutrino data from the Fermi bubbles. Right panel: Expected event rates in HAWC and IceCube from Fermi bubbles in hadronic model. Figures taken from Lunardini et al. (2015).
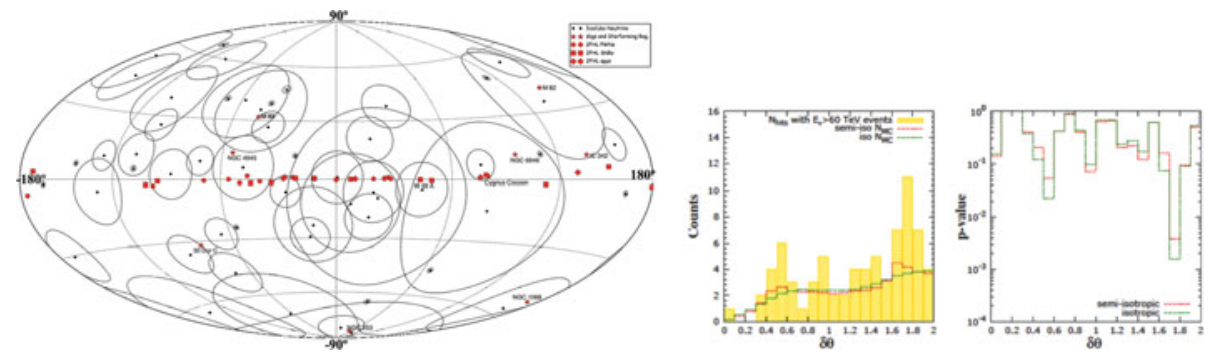

Figure 2. Left panel: Arrival directions of the 53 IceCube HESE neutrinos with their uncertainty ellipses in Galactic coordinates. Also shown are 33 Galactic objects from the Fermi-LAT 2FHL catalog. Right panel: Angular correlation between the HESE neutrinos and the 2FHL Galactic objects. Figures taken from Moharana \& Razzaque (2016).

We have performed a cross correlation study for the highest energy gamma ray sources in the Fermi 2FHL catalog (Ackermann et al. (2016)) that includes 33 Galactic sources such as supernova remnants and pulsar wind nebulae, which are associated to star formation. These Galactic sources show a significant correlation with the HESE neutrinos, giving p-value (pre-trial) 0.017 for all neutrino energies and a lower p-value (pre-trial) 0.0016 for neutrinos with energy greater than $60 \mathrm{TeV}$. The post-trial p-value is 0.06 for these 2FHL Galactic sources.

\section{References}

Aartsen, M. G., Ackermann, M., Adams, J., et al. 2014, Phys. Rev. Lett., 113, 101101

Ackermann, M., Albert, A., Atwood, W. B., et al. 2014, ApJ, 793, 64

Ackermann, M., Ajello, M., Atwood, W. B., et al. 2016, ApJS, 222, 5

Crocker, R. M. \& Aharonian, F. 2011, Phys. Rev. Lett., 106, 101102

Emig, K., Lunardini, C., \& Windhorst, R. 2015, JCAP, 12, 029

Goodman, J. 2016, in this proceedings

Kopper, C. 2015, in PoS, ICRC2015, 1081

Lunardini, C., Razzaque, S., Theodoseau, K. T., \& Yang, L. 2014, Phys. Rev. D, 90, 023016

Lunardini, C. \& Razzaque, S. 2012, Phys. Rev. Lett., 108, 221102

Lunardini, C., Razzaque, S., \& Yang, L. 2015, Phys. Rev. D, 92, 021301

Moharana, R. \& Razzaque, S. 2015, JCAP, 8, 014

Moharana, R. \& Razzaque, S. 2016, arXiv:1606.04420

Razzaque, S. 2013, Phys. Rev. D, 88, 081302

Su, M., Slatyer, T. R., \& Finkbeiner, D. P. 2010, ApJ, 724, 1044 